\title{
On Radial Two-Species Onsager Vortices Near the Critical Temperature
}

\author{
TONIA RICCIARDI \& RYO TAKAHASHI
}

\begin{abstract}
We compare two mean field equations describing hydrodynamic turbulence in equilibrium, which are derived under a deterministic vs. stochastic assumption on the variable vortex intensity distribution. Mathematically, such equations correspond to non-local Liouville type problems, and the critical temperature corresponds to the optimal Moser-Trudinger constant. We consider the radial case and we assume that the inverse temperature is near its critical value. Under these assumptions we show that, unlike previously existing results, the qualitative properties of the solution set in the deterministic case is more similar to the single vortex intensity case than the stochastic case. Some new variational interpretations of the value-explicit values of the critical temperature are also provided.
\end{abstract}

\section{INTRODUCTION}

In recent years, in the context of the statistical mechanics of two-dimensional point vortices, as introduced in the pioneering work of Onsager [26], several nonlocal elliptic equations, including an exponential type nonlinearity, have been derived in order to take into account variable vortex intensities. (See, e.g., [7,39] and the references therein.)

In particular, assuming that the distribution of the (normalized) vortex intensity $\alpha$ is determined by a probability distribution $\mathcal{P}(\mathrm{d} \alpha), \alpha \in[-1,1]$, the following multi-species Boltzmann-Poisson type problem was derived in [35] (see 
also $[11,37])$ :

$$
\begin{cases}-\Delta v=\lambda \int_{[-1,1]} \alpha \frac{e^{\alpha v}}{\int_{\Omega} e^{\alpha v} \mathrm{~d} x} \mathcal{P}(\mathrm{d} \alpha) & \text { in } \Omega, \\ v=0 & \text { on } \partial \Omega .\end{cases}
$$

Here, $\Omega \subset \mathbb{R}^{2}$ is a smooth bounded domain, $v$ denotes the stream function of the turbulent Euler flow, and $\lambda>0$ is a physical constant related to the inverse temperature. It should be mentioned that problems of the form (1.1) also appear in the description of equilibrium states for Brownian gases and chemotaxis systems with different types of particle species, subject to the conserved mass constraint for each species (see $[8,9,14,18]$ and the references therein), as well as in affine geometry (see [16]). Here, we are particularly interested in the "two-species, positive case," where $\mathcal{P}$ is given by

$$
\mathcal{P}(\mathrm{d} \alpha)=\tau \delta_{1}(\mathrm{~d} \alpha)+(1-\tau) \delta_{\gamma}(\mathrm{d} \alpha)
$$

for some $\tau, \gamma \in(0,1)$. Such an assumption corresponds to the physically relevant case where the point vortices have intensity 1 or $\gamma$, in proportion $\tau$ and $1-\tau$, respectively, and are all equally oriented. For such a choice of $\mathcal{P}$, problem (1.1) reduces to the problem

$$
\begin{cases}-\Delta v=\lambda\left(\tau \frac{e^{v}}{\int_{\Omega} e^{v} \mathrm{~d} x}+(1-\tau) \gamma \frac{e^{\gamma v}}{\int_{\Omega} e^{\gamma v} \mathrm{~d} x}\right) & \text { in } \Omega, \\ v=0 & \text { on } \partial \Omega .\end{cases}
$$

On the other hand, the following problem, formally similar to (1.1), was derived in [24] in order to describe stationary turbulent flows under a "stochastic" assumption on the vorticity distribution:

$$
\begin{cases}-\Delta v=\lambda \frac{\int_{[-1,1]} \alpha e^{\alpha v} \mathcal{P}(\mathrm{d} \alpha)}{\iint_{\Omega \times[-1,1]} e^{\alpha v} \mathrm{~d} x \mathcal{P}(\mathrm{d} \alpha)} & \text { in } \Omega, \\ v=0 & \text { on } \partial \Omega .\end{cases}
$$


In this case, it is assumed that the vortex intensities $\alpha \in[-1,1]$ of each point vortex are independent identically distributed random variables with probability distribution $\mathcal{P}(\mathrm{d} \alpha)$. In the "two-species case" where $\mathcal{P}$ is given by (1.2), problem (1.4) takes the form

$$
\begin{cases}-\Delta v=\lambda \frac{\tau e^{v}+(1-\tau) \gamma e^{\gamma v}}{\int_{\Omega}\left\{\tau e^{v}+(1-\tau) e^{\gamma v}\right\} \mathrm{d} x} & \text { in } \Omega, \\ v=0 & \text { on } \partial \Omega .\end{cases}
$$

Note that we can also view Problem (1.4) as the stationary state of a multi-species Boltzmann-Poisson evolution system, where the masses of the individual population species are allowed to vary, provided the total population mass is conserved (see [18]).

In the special case $\mathcal{P}(\mathrm{d} \alpha)=\delta_{1}(\mathrm{~d} \alpha)$, equation (1.1) and equation (1.4) both reduce to the well-understood single-species Boltzmann-Poisson system, which is also known in the mathematical literature as the as "standard" mean field equation

$$
\begin{cases}-\Delta v=\lambda \frac{e^{v}}{\int_{\Omega} e^{v} \mathrm{~d} x} & \text { in } \Omega, \\ v=0 & \text { on } \partial \Omega,\end{cases}
$$

which has been extensively analyzed in view of its applications in biology, differential geometry, and physics. In the context of turbulence, problem (1.6) was rigorously derived and analyzed in [6]. (See [17, 20,21,39] and the references therein for results concerning problem (1.6).)

In recent years, a considerable effort has been devoted to compare qualitative properties of the solutions to problem (1.3) and problem (1.5) (see [16, 22, 25, $30-32,34])$. In $[22,33,34]$ it is shown that problem (1.5) may be viewed as a perturbation of (1.6), and in particular it shares analogous mass quantization properties and an optimal Moser-Trudinger constant (which physically corresponds to the critical temperature) as (1.6), independently of $\mathcal{P}$ (see also [39]). On the other hand, in $[13,16,25,30]$ it is shown that the blow-up masses and the optimal Moser-Trudinger constant for (1.3) significantly depend on $\mathcal{P}$, and that (1.3) is not in general a perturbation of (1.6), with the exception of the case where the probability measure $\mathcal{P}(\mathrm{d} \alpha)$ is sufficiently "close" to the Dirac mass $\delta_{1}(\mathrm{~d} \alpha)$ (see [32]). Sign-changing cases were considered in [15, 17, 27, 28,31].

Our aim in this note is to exhibit a first situation where the deterministic problem (1.3) behaves more similarly to the "standard" problem (1.6) than the stochastic problem (1.5). More precisely, we shall show that when $\Omega$ is a disc and $\mathcal{P}$ is of the form (1.2), the critical temperature for (1.3) determines a threshold for the existence of solutions, exactly as it happens for (1.6), independently of $\tau, \gamma$. On the other hand, the bifurcation diagram of the solution set near the critical 
temperature for (1.5) significantly depends on $\tau, \gamma$, and does not necessarily determine a threshold for the existence of solutions. We expect similar properties to hold true for probability measures $\mathcal{P}$ more general than (1.2). However, the corresponding computations become significantly more complicated in the three mass case, as may be seen in [40], in a different context, so that a different approach appears to be necessary.

This note is organized as follows. In the following section, we state our main results, namely Theorem 2.3 and Theorem 2.4 below. Several preliminary properties necessary to the proofs are derived in Section 3. The proof of Theorem 2.3 is provided in Section 4, and the proof of Theorem 2.4 is provded in Section 5. Finally, some remarks on the explicit value of the critical temperature for the deterministic problem (1.3), namely the constant $\bar{\lambda}_{\tau, \gamma}$ defined in (2.4) below, are provided in Appendix A.

\section{STATEMENT OF THE MAIN Results}

We first recall the variational formulations of problem (1.1) and problem (1.4), given by the functionals

$$
\begin{array}{ll}
J_{\lambda, \mathcal{P}}^{(d)}(v)=\frac{1}{2} \int_{\Omega}|\nabla v|^{2}-\lambda \int_{[-1,1]}\left[\ln \int_{\Omega} e^{\alpha v} \mathrm{~d} x\right] \mathcal{P}(\mathrm{d} \alpha), & v \in H_{0}^{1}(\Omega), \\
J_{\lambda, \mathcal{P}}^{(s)}(v)=\frac{1}{2} \int_{\Omega}|\nabla v|^{2}-\lambda \ln \left[\iint_{\Omega \times[-1,1]} e^{\alpha v} \mathrm{~d} x \mathcal{P}(\mathrm{d} \alpha)\right], & v \in H_{0}^{1}(\Omega),
\end{array}
$$

respectively. For $\mathcal{P}(\mathrm{d} \alpha)=\delta_{1}(\mathrm{~d} \alpha),(2.1)$ and (2.2) reduce to the variational functional for (1.6), given by

$$
I_{\lambda}(v)=\frac{1}{2} \int_{\Omega}|\nabla v|^{2} \mathrm{~d} x-\lambda \ln \int_{\Omega} e^{v} \mathrm{~d} x, \quad v \in H_{0}^{1}(\Omega) .
$$

In view of the Moser-Trudinger inequality [23, 41]

$$
\int_{\Omega} e^{4 \pi v^{2} / \int_{\Omega}|\nabla v|^{2} \mathrm{~d} x} \leq C_{M T}|\Omega| \quad \text { for all } v \in H_{0}^{1}(\Omega) \backslash\{0\},
$$

where the constant $C_{M T}>0$ does not depend on the domain $\Omega$ and where the constant $4 \pi$ is best possible, using the inequality $a b \leq\left(a^{2}+b^{2}\right) / 2$ we derive

$$
v \leq \frac{4 \pi v^{2}}{\int_{\Omega}|\nabla v|^{2} \mathrm{~d} x}+\frac{1}{16 \pi} \int_{\Omega}|\nabla v|^{2} \mathrm{~d} x .
$$

It follows that

$$
\int_{\Omega} e^{v} \mathrm{~d} x \leq C_{M T}|\Omega| e^{(1 / 16 \pi) \int_{\Omega}|\nabla v|^{2} \mathrm{~d} x} \quad \text { for all } v \in H_{0}^{1}(\Omega) .
$$


In particular, it follows that

$$
\inf _{H_{0}^{1}(\Omega)} I_{\lambda}>-\infty \quad \text { if and only if } \lambda \leq 8 \pi,
$$

and that for all $\lambda<8 \pi$ a solution for (1.6) may be obtained by direct minimization of $I_{\lambda}$. The limit value $\lambda=8 \pi$ corresponds to the "critical temperature" for the single species case $\mathcal{P}(\mathrm{d} \alpha)=\delta_{1}(\mathrm{~d} \alpha)$. Such a solution is also unique $[2,38]$. On the other hand, if $\Omega$ is a disc, then a Pohozaev identity argument, as may be found, for example, in [3] (or, alternatively, the explicit form of the solutions; see [1]) implies that problem (1.6) does not admit solutions when $\lambda \geq 8 \pi$. Such existence/non-existence properties were employed in an essential way in [19] to establish the biologically relevant finite-time blow-up of solutions to the corresponding evolution problem for (1.6).

Motivated by the recent results in [18], our aim in this note is to investigate similar properties for (1.3) and (1.5). In order to state our results precisely, we first recall the corresponding extensions of the "optimal Moser-Trudinger inequality" (2.3) to the functionals $J_{\lambda, \mathcal{P}}^{(d)}$ and $J_{\lambda, \mathcal{P}}^{(s)}$ as in $[30,33,39]$.

Let $\bar{\lambda}_{\mathcal{P}}^{(d)}$ be defined by

$$
\bar{\lambda}_{\mathcal{P}}^{(d)}:=8 \pi \inf \left\{\frac{\mathcal{P}\left(K_{ \pm}\right)}{\left(\int_{K_{ \pm}} \alpha \mathcal{P}(\mathrm{d} \alpha)\right)^{2}}: K_{ \pm} \subset I_{ \pm}, \mathcal{P}\left(K_{ \pm}\right)>0\right\},
$$

where $I_{+}:=[0,1], I_{-}:=[-1,0)$ and where $K_{ \pm}$are Borel sets. We say that $\mathcal{P}$ is discrete if $\mathcal{P}=\sum_{i=1}^{m} \boldsymbol{T}_{i} \delta_{\gamma_{i}}$ for some $m \in \mathbb{N}, \boldsymbol{T}_{i} \in(0,1], \sum_{i=1}^{m} \boldsymbol{T}_{i}=1$, $\gamma_{i} \in[-1,1]$. The following optimal conditions for boundedness from below of $J_{\lambda, \mathcal{P}}^{(d)}$ and $J_{\lambda, \mathcal{P}}^{(s)}$ hold true.

Proposition 2.1 ([30,33,36]). It holds that

(i) $\inf _{H_{0}^{1}(\Omega)} J_{\lambda, \mathcal{P}}^{(d)}>-\infty$ if $\lambda<\bar{\lambda}_{\mathcal{P}}^{(d)}$ and $\inf _{H_{0}^{1}(\Omega)} J_{\lambda, \mathcal{P}}^{(d)}=-\infty$ if $\lambda>\bar{\lambda}_{\mathcal{P}}^{(d)}$. Moreover, if $\mathcal{P}$ is discrete, then $\left.\inf _{H_{0}^{1}(\Omega)} J_{\lambda, \mathcal{P}}^{(d)}\right|_{\lambda=\bar{\lambda}_{\mathcal{P}}^{(d)}}>-\infty$.

(ii) It holds that $\inf _{H_{0}^{1}(\Omega)} J_{\lambda, \mathcal{P}}^{(s)}>-\infty$ if and only if $\lambda \leq 8 \pi$.

In the special case where $\mathcal{P}$ is of the two-species form (1.2), the functional $J_{\lambda, \mathcal{P}}^{(d)}$ takes the form

$J_{\lambda}^{(\mathrm{d})}(v)=\frac{1}{2} \int_{\Omega}|\nabla v|^{2} \mathrm{~d} x-\lambda \tau \ln \int_{\Omega} e^{v} \mathrm{~d} x-\lambda(1-\tau) \ln \int_{\Omega} e^{\gamma v} \mathrm{~d} x, \quad v \in H_{0}^{1}(\Omega)$,

corresponding to the variational functional for (1.3). In view of Proposition 2.1 (i), the resulting Moser-Trudinger inequality for $J_{\lambda}^{(\mathrm{d})}$ is given by

$$
\inf _{H_{0}^{1}(\Omega)} J_{\lambda}^{(\mathrm{d})}>-\infty \quad \text { if and only if } \quad \lambda \leq \bar{\lambda}_{\tau, \gamma}
$$


where $\bar{\lambda}_{\tau, \gamma}=\left.\bar{\lambda}_{\mathcal{P}}^{(d)}\right|_{\mathcal{P}=\tau \delta_{1}+(1-\tau) \delta_{\gamma}}$ is given by

$$
\begin{aligned}
\bar{\lambda}_{\tau, \gamma} & =8 \pi \min \left\{\frac{1}{\{\tau+(1-\tau) \gamma\}^{2}}, \frac{1}{\tau}\right\} \\
& = \begin{cases}\frac{8 \pi}{\tau}, & \text { if } 0<\gamma \leq \frac{\sqrt{\tau}}{1+\sqrt{\tau}}, \\
\frac{8 \pi}{\{\tau+(1-\tau) \gamma\}^{2}}, & \text { if } \frac{\sqrt{\tau}}{1+\sqrt{\tau}}<\gamma<1 .\end{cases}
\end{aligned}
$$

Remark 2.2. Roughly speaking, the case $\bar{\lambda}_{\tau, \gamma}=8 \pi / \tau$ corresponds to the case where the term $e^{\gamma v}$ is a perturbation of the term $e^{v}$, whereas the case $\bar{\lambda}_{\tau, \gamma}=$ $8 \pi /\{\tau+(1-\tau) \gamma\}^{2}$ corresponds to the situation where both terms $e^{v}, e^{\gamma v}$ influence the Moser-Trudinger constant. When $\gamma \geq \frac{1}{2}$, straightforward considerations involving "Liouville bubbles" imply that both nonlinearities should concentrate along a blowing-up sequence, so that the second case is natural. On the other hand, the threshold value satisfies $\sqrt{T} /(1+\sqrt{T})<\frac{1}{2}$ for all $\tau \in(0,1)$. So, in the interval $\left(\sqrt{T} /(1+\sqrt{T}), \frac{1}{2}\right)$ the functional is also influenced by the lower-order term, although it does not blow up along the sequence of minimizers. See Appen$\operatorname{dix}$ A for some considerations which motivate the role of $\tau$ in this context.

In particular, for all $\lambda<\bar{\lambda}_{\tau, \gamma}$ there exists a solution to (1.3) corresponding to the minimum of $J_{\lambda}^{(\mathrm{d})}$. Our first result states that if $\Omega$ is the unit disc, similarly as for the "standard" case $\tau=1$, the optimal Moser-Trudinger constant $\bar{\lambda}_{\tau, \gamma}$ provides a sharp threshold for the existence of solutions to (1.3), as follows.

Theorem 2.3. Suppose $\Omega=B_{1}(0)$. For any $\tau, \gamma \in(0,1)$, a solution to (1.3) exists if and only if $\lambda<\bar{\lambda}_{\tau, \gamma}$.

On the other hand, the variational functional for (1.5) is given by

$J_{\lambda}^{(\mathrm{s})}(v)=\frac{1}{2} \int_{\Omega}|\nabla v|^{2} \mathrm{~d} x-\lambda \ln \left(\tau \int_{\Omega} e^{v} \mathrm{~d} x+(1-\tau) \int_{\Omega} e^{\gamma v} \mathrm{~d} x\right), \quad v \in H_{0}^{1}(\Omega)$,

corresponding to the functional $J_{\lambda, \mathcal{P}}^{(s)}$ with $\mathcal{P}$ satisfying the two-species form (1.2). In view of Proposition 2.1 (ii) (see also [39]), we have that the optimal MoserTrudinger constant for $J_{\lambda}^{(\mathrm{s})}$ is independent of $\tau, \gamma$ :

$$
\inf _{H_{0}^{1}(\Omega)} J_{\lambda}^{(\mathrm{s})}>-\infty \quad \text { if and only if } \quad \lambda \leq 8 \pi .
$$

Our next result shows that, on the contrary, the solution set of (1.5) for $\lambda$ near $8 \pi$ significantly depends on the parameters $\tau, \gamma$. Indeed, for fixed $\tau, \gamma$, let

$$
\lambda_{\tau, \gamma}^{*}:=\sup \{\lambda>0:(1.5) \text { admits a solution }\} .
$$


By direct minimization of $J_{\lambda}^{(\mathrm{s})}$ and the Moser-Trudinger inequality in [33], we have $\lambda_{\tau, \gamma}^{*} \geq 8 \pi$. If $\Omega$ is the unit disc, for small values of $\tau$, the strict inequality holds true.

Theorem 2.4. Suppose $\Omega=B_{1}(0)$. For every fixed $\gamma \in(0,1)$ there exists $\underline{\tau}_{\gamma} \in(0,1)$ such that $\lambda_{\tau, \gamma}^{*}>8 \pi$ for all $\boldsymbol{T} \in\left(0, \underline{\tau}_{\gamma}\right)$.

In particular, Theorem 2.3 and Theorem 2.4 imply that the bifurcation diagram for the deterministic problem (1.3) is qualitatively more similar to the bifurcation diagram for (1.6) than the corresponding diagram for (1.5). This appears to be the first situation where (1.3) is more similar to (1.6) than (1.5). A more precise description of $\underline{\underline{I}}_{\gamma} \in(0,1)$ requires different arguments and will be addressed in a forthcoming paper.

Henceforth, we assume $\Omega=B_{1}(0)$.

Notation. We omit the integration variable when it is clear from the context. For $\sigma>0$ we denote by $B_{\sigma}$ the disc centered at 0 with radius $\sigma$. We denote by $C>0$ a general constant whose actual value is allowed to vary from line to line.

\section{PRELIMINARIES}

We begin by establishing relations between the non-local mean field problems (1.3) and (1.5) defined on $B_{1}$, namely, problem

$$
\begin{cases}-\Delta v=\lambda\left(\tau \frac{e^{v}}{\int_{B_{1}} e^{v}}+(1-\tau) \gamma \frac{e^{\gamma v}}{\int_{B_{1}} e^{\gamma v}}\right) & \text { in } B_{1}, \\ v=0 & \text { on } \partial B_{1},\end{cases}
$$

and problem

$$
\begin{cases}-\Delta v=\lambda \frac{\tau e^{v}+(1-\tau) \gamma e^{\gamma v}}{\int_{B_{1}}\left\{\tau e^{v}+(1-\tau) e^{\gamma v}\right\}} & \text { in } B_{1}, \\ v=0 & \text { on } \partial B_{1},\end{cases}
$$

and the following local problem defined on whole space:

$$
\left\{\begin{array}{l}
-\Delta z=e^{z}+e^{y z} \\
z(0)=\max _{\mathbb{R}^{2}} z=\alpha \in \mathbb{R}, \\
\int_{\mathbb{R}^{2}}\left\{e^{z}+e^{\gamma z}\right\}=m(\alpha)<+\infty,
\end{array}\right.
$$

which is a special case of the cosmic string problem considered in [10,29]. We note that solutions to (3.1) and (3.2) are radially symmetric [12].

Indeed, our aim in this section is to establish the following properties. 
Proposition 3.1 (Reduction of the deterministic problem to (3.3)). Let $v$ be a solution to problem (3.1). Then, the function $z$ defined by

$$
z(y)=v\left(\frac{y}{\sigma}\right)+\frac{1}{1-\gamma} \ln \left[\frac{\tau}{(1-\tau) \gamma} \frac{\int_{B_{1}} e^{\gamma v}}{\int_{B_{1}} e^{v}}\right], \quad y \in B_{\sigma}
$$

where $\sigma$ is defined by

$$
\sigma^{2(1-\gamma)}=\frac{\lambda^{1-\gamma}(1-\tau) \gamma}{\tau^{\gamma}} \frac{\left(\int_{B_{1}} e^{v}\right)^{\gamma}}{\int_{B_{1}} e^{\gamma v}}
$$

is a solution to the problem

$$
\left\{\begin{array}{l}
-\Delta z=e^{z}+e^{\gamma z} \quad \text { in } B_{\sigma}, \\
\int_{B_{\sigma}} e^{z}=\lambda \tau, \\
\int_{B_{\sigma}} e^{\gamma z}=\lambda(1-\tau) \gamma,
\end{array}\right.
$$

satisfying

$$
z(0)=\max _{\mathbb{R}^{2}} z=v(0)+\frac{1}{1-\gamma} \ln \left[\frac{\tau}{(1-\tau) \gamma} \frac{\int_{B_{1}} e^{\gamma v}}{\int_{B_{1}} e^{v}}\right] .
$$

Similarly, we also have the following result.

Proposition 3.2 (Reduction of the stochastic problem to (3.3)). Let $v$ be a solution to problem (3.2). Then, the function $z$ defined by

$$
z(y)=v\left(\frac{y}{\sigma}\right)+\frac{1}{1-\gamma} \ln \left[\frac{\tau}{(1-\tau) \gamma}\right], \quad y \in B_{\sigma}
$$

where $\sigma$ is defined by

$$
\sigma^{2(1-\gamma)}=\frac{\lambda^{1-\gamma}(1-\tau) \gamma}{\tau^{\gamma}}\left(\frac{1}{\int_{B_{1}}\left\{\tau e^{v}+(1-\tau) e^{\gamma v}\right\}}\right)^{1-\gamma}
$$


is a solution to the problem

$$
\begin{cases}-\Delta z=e^{z}+e^{\gamma z}, & \text { in } B_{\sigma}, \\ z=\frac{1}{1-\gamma} \ln \frac{\tau}{(1-\tau) \gamma}, & \text { on } \partial B_{\sigma}, \\ \int_{B_{\sigma}}\left\{e^{z}+\frac{e^{\gamma z}}{\gamma}\right\}=\lambda, & \end{cases}
$$

satisfying

$$
z(0)=\max _{\mathbb{R}^{2}} z=v(0)+\frac{1}{1-\gamma} \ln \left[\frac{T}{(1-\tau) \gamma}\right] .
$$
results.

Once we are reduced to problem (3.3), we may use the following known

Lemma 3.3 ([42]). For any $a, b>0$ and for any $\alpha \in \mathbb{R}$, the initial value problem

$$
\left\{\begin{array}{l}
-\left(\eta^{\prime \prime}+\frac{1}{r} \eta^{\prime}\right)=a e^{\eta}+b e^{\gamma \eta}, \quad r>0 \\
\eta(0)=\alpha \\
\eta^{\prime}(0)=0
\end{array}\right.
$$

admits a unique globally defined solution for any $\alpha \in \mathbb{R}$. Moreover, there exists $\beta>2 / \gamma$ such that

$$
\lim _{r \rightarrow+\infty} \frac{\eta(r)}{\ln r}=-\beta
$$

Proof. In order to obtain the local existence of solutions to (3.6), we rewrite the problem in the form $\eta^{\prime}=F(r, \eta), \eta(0)=\alpha$, where

$$
F(r, \eta)= \begin{cases}-\frac{1}{r} \int_{0}^{r} s f(\eta(s)) \mathrm{d} s, & \text { if } r \geq 0, \\ 0 & \text { if } r<0,\end{cases}
$$

and $f(t)=a e^{t}+b e^{\gamma t}$. It is readily checked that $F$ is continuous and locally Lipschitz continuous with respect to $\eta$. Hence, standard ODE theory yields the asserted local existence and uniqueness. Now, the global existence and the asymptotic behavior follow by Lemma 5.1 and Lemma 5.2 in [42] (see also [4]).

The sharp range of admissible values of the constant $\beta$ in Lemma 3.3 was determined in [29].

Lemma 3.4 ([10,29]). For every $\alpha \in \mathbb{R}$ there exists a unique solution to (3.3). Such a solution is radially symmmetric with respect to 0 and strictly decreasing with 
respect to $r=|x|$. Moreover, $m(\alpha)$ is strictly decreasing with respect to $\alpha \in \mathbb{R}$ and it satisfies

$$
\begin{aligned}
& \lim _{\alpha \rightarrow+\infty} m(\alpha)=8 \pi \max \left\{1, \frac{1-\gamma}{\gamma}\right\}= \begin{cases}8 \pi & \text { if } \frac{1}{2} \leq \gamma<1, \\
8 \pi \frac{1-\gamma}{\gamma} & \text { if } 0<\gamma<\frac{1}{2},\end{cases} \\
& \lim _{\alpha \rightarrow-\infty} m(\alpha)=\frac{8 \pi}{\gamma} .
\end{aligned}
$$

We set

$$
m_{1}(\alpha):=\int_{\mathbb{R}^{2}} e^{z}, \quad m_{\gamma}(\alpha):=\int_{\mathbb{R}^{2}} e^{\gamma z},
$$

where $z$ is a solution to (3.3). We also need an "energy identity" from [10].

Lemma 3.5 ([10]). The following relation holds true:

$$
\left(m_{1}(\alpha)+m_{\gamma}(\alpha)\right)^{2}=8 \pi\left(m_{1}(\alpha)+\frac{m_{\gamma}(\alpha)}{\gamma}\right) \quad \text { for any } \alpha \in \mathbb{R} .
$$

Remark 3.6. From Lemma 3.4 and Lemma 3.5 it follows in particular that

$$
m_{1}(\alpha)<8 \pi \quad \text { for all } \alpha \in \mathbb{R} .
$$

In order to establish Proposition 3.1 and Proposition 3.2, we begin by observing the following general fact.

Lemma 3.7. Let $v$ be a solution to the problem

$$
\begin{cases}-\Delta v=a e^{v}+b e^{\gamma v} & \text { in } B_{1}, \\ v=0 & \text { on } \partial B_{1},\end{cases}
$$
where $a, b>0$. Let $z(y):=v\left(\frac{y}{\sigma}\right)+\ln \left(\frac{a}{\sigma^{2}}\right)$, where $y \in B_{\sigma}$, with $\sigma>0$
defined by

$$
\sigma^{2(1-\gamma)}=\frac{b}{a^{\gamma}} .
$$

Then, $z$ is a solution to the problem

$$
\begin{cases}-\Delta z=e^{z}+e^{\gamma z} & \text { in } B_{\sigma} \\ z=\frac{1}{1-\gamma} \ln \frac{a}{b} & \text { on } \partial B_{\sigma} \\ \int_{B_{\sigma}} e^{z(y)} \mathrm{d} y=\int_{B_{1}} a e^{v(x)} \mathrm{d} x, & \\ \int_{B_{\sigma}} e^{\gamma z(y)} \mathrm{d} y=\int_{B_{1}} b e^{\gamma v(x)} \mathrm{d} x . & \end{cases}
$$


Proof. The function $w:=v+\ln a$ satisfies

$$
\begin{cases}-\Delta w=e^{w}+\frac{b}{a^{\gamma}} e^{\gamma w} & \text { in } B_{1}, \\ w=\ln a & \text { on } \partial B_{1}, \\ \int_{B_{1}} e^{w}=\int_{B_{1}} a e^{v} & \\ \int_{B_{1}} \frac{b}{a^{\gamma}} e^{\gamma w}=\int_{B_{1}} b e^{\gamma v} . & \end{cases}
$$

For $\sigma>0$ we rescale

$$
z(y):=w\left(\frac{y}{\sigma}\right)+2 \ln \frac{1}{\sigma}=v\left(\frac{y}{\sigma}\right)+\ln \left(\frac{a}{\sigma^{2}}\right), \quad y \in B_{\sigma} .
$$

Then, $z$ satisfies

$$
\begin{cases}-\Delta z=e^{z}+\sigma^{-2(1-\gamma)} \frac{b}{a^{\gamma}} e^{\gamma z} & \text { in } B_{\sigma}, \\ z=\ln \frac{a}{\sigma^{2}} & \text { on } \partial B_{\sigma}, \\ \int_{B_{\sigma}} e^{z(y)} \mathrm{d} y=\int_{B_{1}} e^{w(x)} \mathrm{d} x, & \\ \int_{B_{\sigma}} \sigma^{-2(1-\gamma)} e^{\gamma z(y)} \mathrm{d} y=\int_{B_{1}} e^{\gamma w(x)} \mathrm{d} x . & \end{cases}
$$

We observe that (3.9) implies that

$$
\frac{a}{\sigma^{2}}=\left(\frac{a}{b}\right)^{1 /(1-\gamma)}
$$

We deduce that if $\sigma$ is defined by (3.9), then $z$ satisfies (3.10), as asserted.

Proof of Proposition 3.1. We apply Lemma 3.7 with

$$
a=\frac{\lambda \tau}{\int_{B_{1}} e^{v}}, \quad b=\frac{\lambda(1-\tau) \gamma}{\int_{B_{1}} e^{\gamma v}} .
$$

Choosing $\sigma$ according to (3.9), that is,

$$
\sigma^{2(1-\gamma)}=\frac{b}{a^{\gamma}}=\frac{\lambda^{1-\gamma}(1-\tau) \gamma}{\tau^{\gamma}} \frac{\left(\int_{B_{1}} e^{v}\right)^{\gamma}}{\int_{B_{1}} e^{\gamma v}},
$$

we obtain, recalling (3.11), that 


$$
\frac{a}{\sigma^{2}}=\left[\frac{\tau}{(1-\tau) \gamma} \frac{\int_{B_{1}} e^{\gamma v}}{\int_{B_{1}} e^{v}}\right]^{1 /(1-\gamma)} .
$$

Thus, the function $z$ defined by (3.4) satisfies the problem

$$
\left\{\begin{array}{l}
-\Delta z=e^{z}+e^{\gamma z} \\
z=\frac{1}{1-\gamma} \ln \left[\frac{\tau}{(1-\tau) \gamma} \frac{\int_{B_{1}} e^{\gamma v}}{\int_{B_{1}} e^{v}}\right] \text { on } \partial B_{\sigma}, \\
\int_{B_{\sigma}} e^{z}=\int_{B_{1}} a e^{v}=\lambda \tau, \\
\int_{B_{\sigma}} e^{\gamma z}=\int_{B_{1}} b e^{\gamma v}=\lambda(1-\tau) \gamma,
\end{array}\right.
$$

and the asserted reduction is established.

Similarly, we establish the reduction of problem (3.2).

Proof of Proposition 3.2. We apply Lemma 3.7 with

$$
a=\frac{\lambda \tau}{\int_{B_{1}}\left\{\tau e^{v}+(1-\tau) e^{\gamma v}\right\}}, \quad b=\frac{\lambda(1-\tau) \gamma}{\int_{B_{1}}\left\{\tau e^{v}+(1-\tau) e^{\gamma v}\right\}} .
$$

Choosing $\sigma>0$ according to (3.9), that is,

$$
\sigma^{2(1-\gamma)}=\frac{b}{a^{\gamma}}=\frac{\lambda^{1-\gamma}(1-\tau) \tau^{-\gamma} \gamma}{\left(\int_{B_{1}}\left\{\tau e^{v}+(1-\tau) e^{\gamma v}\right\}\right)^{1-\gamma}},
$$

we find, recalling (3.11), that

$$
\frac{a}{\sigma^{2}}=\left[\frac{\tau}{(1-\tau) \gamma}\right]^{1 /(1-\gamma)} .
$$

Moreover,

$$
\begin{gathered}
\int_{B_{\sigma}} e^{z}=\int_{B_{1}} a e^{v}=\frac{\lambda \tau \int_{B_{1}} e^{v}}{\int_{B_{1}}\left\{\tau e^{v}+(1-\tau) e^{\gamma v}\right\}}, \\
\int_{B_{\sigma}} e^{\gamma z}=\int_{B_{1}} b e^{\gamma v}=\frac{\lambda(1-\tau) \gamma \int_{B_{1}} e^{\gamma v}}{\int_{B_{1}}\left\{\tau e^{v}+(1-\tau) e^{\gamma v}\right\}} .
\end{gathered}
$$


Then, the function $z$ defined by (3.5) is a solution to the problem

$$
\begin{cases}-\Delta z=e^{z}+e^{\gamma z}, & \text { in } B_{\sigma}, \\ z=\frac{1}{1-\gamma} \ln \frac{\tau}{(1-\tau) \gamma}, & \text { on } \partial B_{\sigma}, \\ \int_{B_{\sigma}} e^{z}+\frac{e^{\gamma z}}{\gamma}=\lambda, & \end{cases}
$$

as asserted.

\section{Proof of Theorem 2.3}

In order to prove Theorem 2.3 we use the following Pohozaev identity. The radial symmetry is used in an essential way in the following arguments.

Lemma 4.1 (Pobozaev identity). The following identity holds true:

$$
-\frac{1}{2} \int_{\partial B_{1}}|\nabla v|^{2} \mathrm{~d} \sigma=-2 \lambda+2 \pi \lambda\left(\frac{\tau}{\int_{B_{1}} e^{v}}+\frac{1-\tau}{\int_{B_{1}} e^{\gamma v}}\right) .
$$

Proof. Multiplying (1.3) by $x \cdot \nabla v$ and integrating, we have

$$
\int_{B_{1}}(x \cdot \nabla v)(-\Delta v) \mathrm{d} x=-\frac{1}{2} \int_{\partial B_{1}}|\nabla v|^{2} \mathrm{~d} \sigma .
$$

Similarly,

$$
\begin{aligned}
\int_{B_{1}}(x \cdot \nabla v) \lambda\left(\tau \frac{e^{v}}{\int_{B_{1}} e^{v}}+(1-\tau) \gamma \frac{e^{\gamma v}}{\int_{B_{1}} e^{\gamma v}}\right) \\
=\lambda \int_{B_{1}} x \cdot\left(\tau \frac{\nabla e^{v}}{\int_{B_{1}} e^{v}}+(1-\tau) \frac{\nabla e^{\gamma v}}{\int_{B_{1}} e^{\gamma v}}\right) \\
=\lambda \int_{\partial B_{1}}\left(\frac{\tau}{\int_{B_{1}} e^{v}}+\frac{1-\tau}{\int_{B_{1}} e^{\gamma v}}\right)-2 \lambda \\
=-2 \lambda+2 \pi \lambda\left(\frac{\tau}{\int_{B_{1}} e^{v}}+\frac{1-\tau}{\int_{B_{1}} e^{\gamma v}}\right)
\end{aligned}
$$


Proof of Theorem 2.3. We prove separately the two cases, corresponding to the two possible values of the Moser-Trudinger constant $\bar{\lambda}_{\tau, \gamma}$ defined in (2.4). Throughout this proof, for the sake of simplicity, we denote $\bar{\lambda}=\bar{\lambda}_{\tau, \gamma}$.

Case 1: $\bar{\lambda}=8 \pi /\{\tau+(1-\tau) \gamma\}^{2}$. Recalling that $\Omega=B_{1}$, and using the Schwarz inequality, we have

$$
\begin{aligned}
\int_{\partial B_{1}}|\nabla v|^{2} \mathrm{~d} \sigma & =\int_{\partial B_{1}}\left|\frac{\partial v}{\partial v}\right|^{2} \mathrm{~d} \sigma \geq \frac{1}{\left|\partial B_{1}\right|}\left(\int_{\partial B_{1}}\left(-\frac{\partial v}{\partial v}\right) \mathrm{d} \sigma\right)^{2} \\
& =\frac{1}{2 \pi}\left(\int_{B_{1}}(-\Delta v) \mathrm{d} x\right)^{2}=\frac{\lambda^{2}}{2 \pi}\{\tau+(1-\tau) \gamma\}^{2} .
\end{aligned}
$$

In view of Lemma 4.1 we deduce that

$$
\begin{aligned}
\lambda & \leq \frac{8 \pi}{\{\tau+(1-\tau) \gamma\}^{2}}\left\{1-\pi\left(\frac{\tau}{\int_{B_{1}} e^{v}}+\frac{1-\tau}{\int_{B_{1}} e^{\gamma v}}\right)\right\} \\
& <\frac{8 \pi}{\{\tau+(1-\tau) \gamma\}^{2}}=\bar{\lambda} .
\end{aligned}
$$

Case 2: $\bar{\lambda}=8 \pi / \tau$. Let $z$ be defined as in Proposition 3.1. By Lemma 3.3 and Lemma 3.4, $z$ coincides in $B_{\sigma}$ with the unique solution $z_{\alpha}$ to (3.3) with $\alpha=\alpha^{*}$ given by

$$
\alpha^{*}=v(0)+\frac{1}{1-\gamma} \ln \frac{\tau \int_{B_{1}} e^{\gamma v}}{(1-\tau) \gamma \int_{B_{1}} e^{v}} .
$$

Consequently, (3.8) assures

$$
\int_{B_{\sigma}} e^{z}=\lambda \tau<\int_{\mathbb{R}^{2}} e^{z_{\alpha^{*}}}=m_{1}\left(\alpha^{*}\right)<8 \pi .
$$

In particular, we derive from the above that

$$
\lambda<\frac{8 \pi}{\tau}=\bar{\lambda} .
$$

Now, Theorem 2.3 is completely established.

\section{Proof of Theorem 2.4}

Our first aim in this section is to establish the following "converse" for Proposition 3.2. It is convenient to define

$$
\beta_{\tau, \gamma}:=\frac{1}{1-\gamma} \ln \frac{\tau}{(1-\tau) \gamma} .
$$


Proposition 5.1. Let $z$ be a solution to (3.3) with $\alpha>\beta_{\tau, \gamma}$. Let $\sigma>0$ be defined by

$$
\left.z\right|_{\partial B_{\sigma}}=\beta_{\tau, \gamma}
$$

and let $a>0$ be defined by

$$
\frac{a}{\sigma^{2}}=e^{\beta_{\tau, \gamma}}=\left[\frac{\tau}{(1-\tau) \gamma}\right]^{1 /(1-\gamma)} .
$$

Then, the function $v$ defined by

$$
v(x)=z(\sigma x)+\ln \frac{\sigma^{2}}{a}=z(\sigma x)-\beta_{\tau, \gamma}
$$

is a solution to problem (3.2) with

$$
\lambda=\int_{B_{\sigma}}\left(e^{z}+\frac{e^{\gamma z}}{\gamma}\right) .
$$

Proof. The function $v$ defined in (5.4) satisfies the equation

$$
-\Delta v=a e^{v}+\sigma^{2(1-\gamma)} a^{\gamma} e^{\gamma v}=\sigma^{2}\left[\frac{a}{\sigma^{2}} e^{v}+\left(\frac{a}{\sigma^{2}}\right)^{\gamma} e^{\gamma v}\right] .
$$

Hence, we obtain from (3.2) the necessary condition

$$
\frac{a}{\sigma^{2} \lambda \tau}=\left(\frac{a}{\sigma^{2}}\right)^{\gamma} \frac{1}{\lambda(1-\tau) \gamma}=\frac{1}{\sigma^{2} \int_{B_{1}}\left\{\tau e^{v}+(1-\tau) e^{\gamma v}\right\}},
$$

from which (5.3) follows. On the other hand, (5.2) is a consequence of the Dirichlet boundary condition for $v$.

Finally, we compute, for $v$ given by (5.4),

$$
\begin{aligned}
\int_{B_{1}} e^{v} \mathrm{~d} x & =\frac{\sigma^{2}}{a} \int_{B_{1}} e^{z(\sigma x)} \mathrm{d} x=\frac{e^{-\beta_{\tau, \gamma}}}{\sigma^{2}} \int_{B_{\sigma}} e^{z}, \\
\int_{B_{1}} e^{\gamma v} & =\left(\frac{\sigma^{2}}{a}\right)^{\gamma} \int_{B_{1}} e^{\gamma z(\sigma x)} \mathrm{d} x=\frac{e^{-\gamma \beta_{\tau, \gamma}}}{\sigma^{2}} \int_{B_{\sigma}} e^{\gamma z} .
\end{aligned}
$$

In view of the elementary identity

$$
\tau e^{-\beta_{\tau, \gamma}}=\left[\frac{(1-\tau) \gamma}{\tau^{\gamma}}\right]^{1 /(1-\gamma)}=(1-\tau) \gamma e^{-\gamma \beta_{\tau, \gamma}},
$$


we may write

$$
\int_{B_{1}}\left\{\tau e^{v}+(1-\tau) e^{\gamma v}\right\}=\sigma^{-2}\left[\frac{(1-\tau) \gamma}{\tau^{\gamma}}\right]^{1 /(1-\gamma)} \int_{B_{\sigma}}\left(e^{z}+\frac{e^{\gamma z}}{\gamma}\right) .
$$

Inserting (5.6)-(5.7) into (5.5), we deduce that

$$
\begin{aligned}
-\Delta v & =\sigma^{2}\left[e^{\beta_{\tau, \gamma}} e^{v}+e^{\gamma \beta_{\tau, \gamma}} e^{\gamma v}\right]=\sigma^{2}\left[\frac{\tau^{\gamma}}{(1-\tau) \gamma}\right]^{1 /(1-\gamma)}\left\{\tau e^{v}+(1-\tau) \gamma e^{\gamma v}\right\} \\
& =\frac{\tau e^{v}+(1-\tau) \gamma e^{\gamma v}}{\int_{B_{1}}\left\{\tau e^{v}+(1-\tau) e^{\gamma v}\right\}} \int_{B_{\sigma}}\left(e^{z}+\frac{e^{\gamma z}}{\gamma}\right) .
\end{aligned}
$$

We deduce that $v$ defined by (5.4) with (5.2) - (5.3) is a solution to problem (3.2) with

$$
\lambda=\int_{B_{\sigma}}\left(e^{z}+\frac{e^{\gamma z}}{\gamma}\right)
$$

as asserted.

In view of Proposition 5.1 it is natural to consider, for fixed $\tau, \gamma \in(0,1)$ and $\alpha>\beta_{\tau, \gamma}$, where $\beta_{\tau, \gamma}$ is defined in (5.1), solution pairs $(z, \sigma)=\left(z_{\gamma}(\alpha), \sigma_{\tau, \gamma}(\alpha)\right)$ where $z_{\gamma}(\alpha)$ is a solution to problem (3.3) and $\sigma_{\tau, \gamma}(\alpha)$ is uniquely defined by (5.2). Correspondingly, we obtain $\Lambda_{\tau, \gamma}(\alpha)$ defined by

$$
\Lambda_{\tau, \gamma}(\alpha)=\int_{B_{\sigma_{\tau, \gamma}(\alpha)}}\left\{e^{z_{\gamma}(\alpha)}+\frac{e^{\gamma z_{\gamma}(\alpha)}}{\gamma}\right\} .
$$

Let

$$
\begin{aligned}
& \mathcal{I}_{\tau, \gamma}:=\{\lambda>0: \text { there exists a solution to }(3.2)\}, \\
& \mathcal{I}_{\tau, \gamma}^{\prime}:=\left\{\Lambda>0: \Lambda=\Lambda_{\tau, \gamma}(\alpha) \text { for some } \alpha>\beta_{\tau, \gamma}\right\} .
\end{aligned}
$$

In view of Proposition 3.2 and Proposition 5.1, $\mathcal{I}_{\tau, \gamma}=\mathcal{I}_{\tau, \gamma}^{\prime}$. Therefore, we are reduced to the study of $\mathcal{I}_{\tau, \gamma}^{\prime}$. Setting $\Lambda_{\tau, \gamma}^{*}:=\sup \mathcal{I}_{\tau, \gamma}^{\prime}$, we have $\lambda_{\tau, \gamma}^{*}=\Lambda_{\tau, \gamma}^{*} \geq 8 \pi$, where $\lambda_{\tau, \gamma}^{*}$ is defined in (2.5).

Proposition 5.2. Let $\tau, \gamma \in(0,1)$ be fixed. The following properties hold true:

(i) Quantities $\sigma_{\tau, \gamma}(\alpha)$ and $\Lambda_{\tau, \gamma}(\alpha)$ are continuous functions of $\alpha \in\left[\beta_{\tau, \gamma},+\infty\right)$, provided that we set $\sigma_{\tau, \gamma}\left(\beta_{\tau, \gamma}\right)=\Lambda_{\tau, \gamma}\left(\beta_{\tau, \gamma}\right)=0$.

(ii) It holds that $\lim _{\alpha \rightarrow+\infty} \sigma_{\tau, \gamma}(\alpha)=0$.

(iii) It holds that $\lim _{\alpha \rightarrow+\infty} \Lambda_{\tau, \gamma}(\alpha)=8 \pi$. 
Proof. Throughout this proof, for simplicity we omit the subscripts $\tau, \gamma$.

Proof of (i). Given that $\alpha \in\left(\beta_{\tau, \gamma},+\infty\right), \sigma(\alpha)$ and $\Lambda(\alpha)$ are uniquely determined by $\beta_{\tau, \gamma}$ and $z(\alpha)$, the unique solution to (3.3), only. Moreover, it is also the unique solution to (3.6) for $a=b=1$. Therefore, the desired continuity follows from the standard ODE theory, since $\beta_{\tau, \gamma}$ is independent of $\alpha$.

Proof of (ii). Let $\alpha_{k} \rightarrow+\infty$. Let $\left(z_{k}, \sigma_{k}\right)$ be the solution pair to (3.3) and (5.2) with $\alpha=\alpha_{k}$ and $\Lambda_{k}$ defined by (5.8). We claim that $\lim _{k \rightarrow+\infty} \sigma_{k}=0$. Indeed, suppose there exists $\sigma_{0}>0$ and a subsequence, still denoted $\sigma_{k}$, such that $\lim _{k \rightarrow+\infty} \sigma_{k}=\sigma_{0}>0$. We use an argument from [5]. Let $\underline{z}_{k}$ be defined as the unique solution to the problem

$$
\begin{cases}-\Delta \underline{z}_{k}=e^{z_{k}}+e^{\gamma z_{k}} & \text { in } B_{\sigma_{0} / 2}, \\ \underline{z}_{k}=\left.z_{k}\right|_{\partial B_{\sigma_{k}}}=\beta_{\tau, \gamma} & \text { on } \partial B_{\sigma_{0} / 2} .\end{cases}
$$

Then, for $k$ sufficiently large, we have $z_{k} \geq \underline{z}_{k} \geq \beta_{\tau, \gamma}$ in $B_{\sigma_{0} / 2}$ in view of the maximum principle. On the other hand, setting $V_{k}(x):=1+e^{-(1-\gamma) z_{k}}$ we may write

$$
\left\{\begin{array}{l}
-\Delta z_{k}=e^{z_{k}}+e^{\gamma z_{k}}=V_{k} e^{z_{k}} \quad \text { in } B_{\sigma_{k}}, \\
\int_{B_{\sigma_{k}}} V_{k} e^{z_{k}} \leq \int_{B_{\sigma_{k}}}\left\{e^{z_{k}}+\frac{e^{\gamma z_{k}}}{\gamma}\right\},
\end{array}\right.
$$

with

$$
\begin{gathered}
\left\|V_{k}\right\|_{L^{\infty}\left(B_{\sigma_{0}}\right)} \leq 1+e^{-(1-\gamma) B_{\tau, \gamma},} \\
z_{k}(0)=\max _{B_{\sigma_{0}}} z_{k} \rightarrow+\infty, \\
\int_{B_{\sigma_{k}}} e^{z_{k}} \leq m_{1}\left(\alpha_{k}\right) \leq 8 \pi, \\
\int_{B_{\sigma_{k}}} e^{\gamma z_{k}} \leq m_{\gamma}\left(\alpha_{k}\right) \leq \frac{8 \pi}{\gamma},
\end{gathered}
$$

where $m_{1}\left(\alpha_{k}\right), m_{\gamma}\left(\alpha_{k}\right)$ are the masses defined in (3.7). In view of an adaptation of the Brezis-Merle alternative, as may be found, for example, in [22,25], we conclude there exist $n \geq 4 \pi$ and $s \geq 0, s \in L^{1}\left(B_{\sigma_{0}}\right)$ such that, up to subsequences,

$$
e^{z_{k}}+e^{\gamma z_{k}}=V_{k} e^{z_{k}} \stackrel{*}{\rightarrow} n \delta_{0}(\mathrm{~d} x)+s(x) \mathrm{d} x .
$$

We deduce that

$$
\underline{z}_{k}(x) \geq \frac{n}{2 \pi} G_{B_{\sigma_{0} / 2}}(x, 0)+\beta_{\tau, \gamma}
$$

where $G_{B_{\sigma_{0} / 2}}(\cdot, \cdot)$ denotes Green's function on $B_{\sigma_{0} / 2}$. Since $n /(2 \pi) \geq 2$, Fatou's lemma implies that

$$
\int_{B_{\sigma_{0} / 2}} e^{z_{k}} \geq \int_{B_{\sigma_{0} / 2}} e^{\underline{\underline{z}} k} \rightarrow+\infty \quad \text { as } k \rightarrow+\infty
$$


a contradiction.

Proof of (iii). In view of Proposition 5.1, setting

$$
\begin{aligned}
\lambda_{k} & =\int_{B_{\sigma_{k}}}\left\{e^{z_{k}}+\frac{e^{\gamma z_{k}}}{\gamma}\right\}=\Lambda_{k}, \\
v_{k}(x) & =z_{k}\left(\sigma_{k} x\right)-\beta_{\tau, \gamma},
\end{aligned}
$$

we obtain a solution $v_{k}$ to problem (3.2) with $\lambda=\lambda_{k}$. Moreover, we have

$$
v_{k}(0)=\alpha_{k}-\beta_{\tau, \gamma} \rightarrow+\infty \text {. }
$$

Therefore, $\left(\lambda_{k}, v_{k}\right)$ is a blowing-up solution sequence for (3.2) with a unique blow-up point located at 0 . Now, in view of the mass quantization results as established in [31,34], we conclude that $\lim _{k \rightarrow+\infty} \lambda_{k}=\lim _{k \rightarrow+\infty} \Lambda_{k}=8 \pi$.

Now, we are ready to prove our main result for (3.2), using Proposition 5.2 and Lemma 3.4.

Proof of Theorem 2.4. Fix $\gamma \in(0,1)$, and observe that $\beta_{\tau, \gamma}$ as defined in (5.1) is monotonically increasing with respect to $\tau \in(0,1)$ and $\lim _{\tau \rightarrow 0^{+}} \beta_{\tau, \gamma}=-\infty$. Hence, for any $\tau_{1}, \tau_{2} \in(0,1)$ with $\tau_{1}<\tau_{2}$ and for any fixed $\alpha \in\left(\beta_{\tau_{2}, \gamma},+\infty\right)$, we have $\sigma_{\tau_{1}, \gamma}(\alpha)>\sigma_{\tau_{2}, \gamma}(\alpha)$, and consequently $\Lambda_{\tau_{1}, \gamma}(\alpha)>\Lambda_{\tau_{2}, \gamma}(\alpha)>0$. Moreover, $\lim _{\tau \rightarrow 0^{+}} \sigma_{\tau, \gamma}(\alpha)=+\infty$.

Let $\alpha_{0} \in \mathbb{R}$ and $z_{0}=z_{0}(x)$ be uniquely defined by the problem

$$
\left\{\begin{array}{l}
-\Delta z_{0}=e^{z_{0}}+e^{\gamma z_{0}}, \\
z_{0}(0)=\max _{\mathbb{R}^{2}} z_{0}=\alpha_{0}, \\
\int_{\mathbb{R}^{2}}\left\{e^{z_{0}}+e^{\gamma z_{0}}\right\}=\frac{8 \pi}{2}\left(1+\frac{1}{\gamma}\right)>8 \pi .
\end{array}\right.
$$

We deduce that $\lim _{\tau \rightarrow 0^{+}} \Lambda_{T, \gamma}\left(\alpha_{0}\right) \geq 8 \pi\left(1+\gamma^{-1}\right) / 2$. In particular, there exists $0<\tau_{0}=\tau_{0}(\gamma) \ll 1$ such that, for all $\tau \in\left(0, \tau_{0}\right)$, we have $\Lambda_{\tau, \gamma}\left(\alpha_{0}\right)>8 \pi$.

\section{APPENDix A. REMARKS ON $\bar{\lambda}_{\tau, \gamma}$}

Our aim in this appendix is to show that the values of $\bar{\lambda}_{\tau, \gamma}$ given in (2.4) may be interpreted in terms of Liouville bubbles. Indeed, for $\delta>0$ let

$$
U_{\delta}(x):=\ln \frac{8 \delta^{2}}{\left(\delta^{2}+|x|^{2}\right)^{2}}, \quad x \in \mathbb{R}^{2}
$$

be the family of "Liouville bubbles," uniquely determined as solutions to the problem

$$
\left\{\begin{array}{l}
-\Delta U=e^{u} \quad \text { in } \mathbb{R}^{2}, \\
\int_{\mathbb{R}^{2}} e^{U}<+\infty .
\end{array}\right.
$$


Let $P U_{\delta}$ be the projection of $U_{\delta}$ on $H_{0}^{1}\left(B_{1}\right)$, namely,

$$
P U_{\delta}(x)=\ln \frac{\left(\delta^{2}+1\right)^{2}}{\left(\delta^{2}+|x|^{2}\right)^{2}} .
$$

Proposition A.1. For every $\gamma \in(0,1)$ there exists some $t_{\gamma}>0$ such that for all $\lambda>\bar{\lambda}_{\tau, \gamma}$, it holds that $J_{\lambda}\left(t_{\gamma} P U_{\delta}\right) \rightarrow-\infty$ as $\delta \rightarrow 0^{+}$.

The following asymptotic behaviors are readily verified.

Lemma A.2. The following expansions hold, as $\delta \rightarrow 0^{+}$:

$$
\begin{aligned}
& \int_{B_{1}}\left|\nabla P U_{\delta}(x)\right|^{2} \mathrm{~d} x=16 \pi \ln \frac{1}{\delta^{2}}+O(1), \\
& \ln \int_{B_{1}} e^{a P U_{\delta}(x)} \mathrm{d} x= \begin{cases}O(1), & \text { if } 0<a<\frac{1}{2}, \\
\ln \ln \frac{1}{\delta^{2}}+O(1), & \text { if } a=\frac{1}{2}, \\
(2 a-1) \ln \frac{1}{\delta^{2}}+O(1), & \text { if } a>\frac{1}{2},\end{cases}
\end{aligned}
$$

for any $a>0$.

In view of Lemma A.2 we may write

$$
J_{\lambda}\left(t P U_{\delta}\right)= \begin{cases}\left\{8 \pi t^{2}-2 \lambda[\tau+(1-\tau) \gamma] t+\lambda\right\} \ln \frac{1}{\delta^{2}}+O(1), & \text { if } t \gamma>\frac{1}{2}, \\ \left(8 \pi t^{2}-2 \lambda \tau t+\lambda \tau\right) \ln \frac{1}{\delta^{2}}+O(1), & \text { if } t \gamma<\frac{1}{2}\end{cases}
$$

For later convenience, we note that

$$
0<\frac{\tau}{1+\tau}<\frac{\sqrt{T}}{1+\sqrt{\tau}}<\frac{1}{2}, \quad \text { for all } 0<\tau<1 .
$$

Lemma A.3. Suppose that $\gamma>\tau /(1+\tau)$. Then, there exists a solution $t=t_{\gamma}$ to the system

$$
\left\{\begin{array}{l}
t \gamma>\frac{1}{2} \\
8 \pi t^{2}-2 \lambda(\tau+(1-\tau) \gamma) t+\lambda<0
\end{array}\right.
$$

provided that

$$
\lambda>\frac{8 \pi}{(\tau+(1-\tau) \gamma)^{2}} .
$$


Proof. By elementary considerations, a necessary condition for a solution $t$ to the second inequality in (A.2) to exist is that

$$
\lambda^{2}(\tau+(1-\tau) \gamma)^{2}-8 \pi \lambda>0,
$$

and moreover $t_{-}<t<t_{+}$where

$$
t_{ \pm}=\frac{\lambda(\tau+(1-\tau) \gamma) \pm \sqrt{\lambda^{2}(\tau+(1-\tau) \gamma)^{2}-8 \pi \lambda}}{8 \pi} .
$$

Hence, we readily derive (A.3), so, assuming that (A.3) holds true, we are left to check compatibility with the first inequality in (A.2). To this end, we observe that in view of (A.3) we have

$$
t_{+}=\frac{\lambda(\tau+(1-\tau) \gamma)+\sqrt{\lambda^{2}(\tau+(1-\tau) \gamma)^{2}-8 \pi \lambda}}{8 \pi}>\frac{1}{\tau+(1-\tau) \gamma} .
$$

In particular, we have that a sufficient condition for $t_{+} \gamma>\frac{1}{2}$ is given by

$$
\frac{\gamma}{\tau+(1-\tau) \gamma}>\frac{1}{2}
$$

The latter inequality is equivalent to $\gamma>\tau /(1+\tau)$, which holds true by assumption. We conclude there exists a solution $t_{y}$ to (A.2) satisfying $0<t_{+}-t_{\gamma} \ll 1$.

Lemma A.4. If $0<\gamma<\frac{1}{2}$, there exists a solution $t_{\gamma}$ to the system

$$
\left\{\begin{array}{l}
\frac{1}{2}<t<\frac{1}{2 \gamma} \\
8 \pi t^{2}-\lambda \tau(2 t-1)<0
\end{array}\right.
$$

provided $\lambda>8 \pi / \tau$.

Proof. By elementary considerations, solutions to the second inequality in (A.4) exist if and only if $(\lambda \tau)^{2}-8 \pi \lambda \tau>0$, which is equivalent to $\lambda>8 \pi / \tau$. Moreover, solutions satisfy $t_{-}<t<t_{+}$where

$$
t_{ \pm}=\frac{\lambda \tau \pm \sqrt{(\lambda \tau)^{2}-8 \pi \lambda \tau}}{8 \pi} .
$$

Thus, in the inequality $\frac{1}{2}<t_{-}<1 /(2 \gamma)$ we can find a sufficient condition for a solution to (A.4) to exist. It is readily checked that the inequality $t_{-}>\frac{1}{2}$ is always satisfied, since we may write

$$
t_{-}=\frac{(\lambda \tau)^{2}-\left[(\lambda \tau)^{2}-8 \pi \lambda \tau\right]}{8 \pi\left(\lambda \tau+\sqrt{\left.(\lambda \tau)^{2}-8 \pi \lambda \tau\right)}\right.}>\frac{1}{2} .
$$


On the other hand, the inequality $t_{-}<1 /(2 \gamma)$ is equivalent to

$$
\sqrt{(\lambda \tau)^{2}-8 \pi \lambda \tau}>\lambda \tau-\frac{4 \pi}{\gamma} .
$$

Therefore, if $\lambda \tau-4 \pi / \gamma<0$, the second inequality holds true. Assuming that $\lambda \tau-4 \pi / \gamma \geq 0$, that is, $\lambda>4 \pi /(\tau \gamma)$, we obtain from above the inequality

$$
\gamma^{2}\left[(\lambda \tau)^{2}-8 \pi \lambda \tau\right]>(\lambda \tau \gamma-4 \pi)^{2} .
$$

We deduce the equivalent condition $\lambda \tau \gamma(1-\gamma)>2 \pi$. Since $\lambda>4 \pi /(\tau \gamma)$, a sufficient condition for the above is $4 \pi(1-\gamma)>2 \pi$, equivalently $\gamma<\frac{1}{2}$.

Now we can prove Proposition A.1.

Proof of Proposition A.1. We first assume that $\gamma \geq \sqrt{\tau} /(1+\sqrt{\tau})$ and therefore $\bar{\lambda}_{\tau, \gamma}=8 \pi /(\lambda \tau \gamma(1-\gamma))^{2}$. In view of (A.1), we have $\sqrt{\tau} /(1+\sqrt{\tau})>\tau /(1+\tau)$, so in particular $\gamma>\tau /(1+\tau)$. We deduce from Lemma A.3 that there exists a solution $t_{y}$ to system (A.2). Now, Lemma A.2 implies that

$$
\begin{aligned}
J_{\lambda}\left(t_{\gamma} P U_{\delta}\right) & =\left\{8 \pi t_{\gamma}^{2}-\lambda\left[\tau\left(2 t_{\gamma}-1\right)+(1-\tau)\left(2 t_{\gamma} \gamma-1\right)\right]\right\} \ln \frac{1}{\delta^{2}}+O(1) \\
& =\left\{8 \pi t_{\gamma}^{2}-2 \lambda(\tau+(1-\tau) \gamma) t_{\gamma}+\lambda\right\} \ln \frac{1}{\delta^{2}}+O(1) \rightarrow-\infty
\end{aligned}
$$

as $\delta \rightarrow 0^{+}$. Hence, the desired asymptotics is established in this case.

Now, we assume $\gamma<\sqrt{\tau} /(1+\sqrt{\tau})$. Then, $\bar{\lambda}_{\tau, \gamma}=8 \pi / \tau$. Since

$$
\frac{\sqrt{T}}{1+\sqrt{T}}<\frac{1}{2}
$$

we may apply Lemma A.4 to conclude that there exists a solution $t_{y}$ to system (A.4). Lemma A.2 yields

$$
J_{\lambda}\left(t_{\gamma} P U_{\delta}\right)=\left\{8 \pi t_{\gamma}^{2}-\lambda \tau\left(2 t_{\gamma}-1\right)\right\} \ln \frac{1}{\delta^{2}}+O(1) \rightarrow-\infty \quad \text { as } \delta \rightarrow 0^{+} .
$$

Now, Proposition A.1 is completely established.

Acknowledgements. The second author was supported by JSPS KAKENHI (grant no. JP16K17627). Thanks is due to the Università di Napoli Federico II, where part of this work was completed, for their kind hospitality. 


\section{REFERENCES}

[1] C. BANDLE, Isoperimetric Inequalities and Applications, Monographs and Studies in Mathematics, vol. 7, Pitman (Advanced Publishing Program), Boston, Mass.-London, 1980. MR572958

[2] D. BARTOLUCCI and C. S. LIN, Existence and uniqueness for mean field equations on multiply connected domains at the critical parameter, Math. Ann. 359 (2014), no. 1-2, 1-44. http://dx. doi.org/10.1007/s00208-013-0990-6. MR3201892

[3] J. W. BEBERNES and A. A. LACEY, Global existence and finite-time blow-up for a class of nonlocal parabolic problems, Adv. Differential Equations 2 (1997), no. 6, 927-953. MR1606347

[4] H. Berestycki, P. L. LiOns, and L. A. Peletier, An ODE approach to the existence of positive solutions for semilinear problems in $\mathbb{R}^{N}$, Indiana Univ. Math. J. 30 (1981), no. 1, 141-157. http://dx.doi.org/10.1512/iumj.1981.30.30012. MR600039

[5] H. BREZIS and F. MERLE, Uniform estimates and blow-up behavior for solutions of $-\Delta u=$ $V(x) e^{u}$ in two dimensions, Comm. Partial Differential Equations 16 (1991), no. 8-9, 12231253. http://dx.doi.org/10.1080/03605309108820797. MR1132783

[6] E. Caglioti, P. L. Lions, C. MARChioro, and M. Pulvirenti, A special class of stationary flows for two-dimensional Euler equations: a statistical mechanics description. II, Comm. Math. Phys. 174 (1995), no. 2, 229-260. MR1362165

[7] P. H. Chavanis, Statistical mechanics of two-dimensional vortices and stellar systems, Dynamics and Thermodynamics of Systems with Long-range Interactions, Les Houches (2002), Lecture Notes in Phys., vol. 602, Springer, Berlin, 2002, pp. 208-289. http://dx.doi.org/10.1007/ 3-540-45835-2_8. MR2008183

[8] Exact diffusion coefficient of self-gravitating Brownian particles in two dimensions, Eur. Phys. J. B 57 (2007), no. 4, 391-409. http://dx.doi.org/10.1140/epjb/ e2007-00187-2. MR2326671

[9] - Virial theorem for Onsager vortices in two-dimensional hydrodynamics, Eur. Phys. J. Plus 127 (2012), no. 159, 27 pp. https://doi.org/10.1140/epjp/i2012-12159-x.

[10] R. M. Chen, Y. GUO, and D. SPIRN, Asymptotic behavior and symmetry of condensate solutions in electroweak theory, J. Anal. Math. 117 (2012), 47-85. http://dx.doi.org/10.1007/ s11854-012-0014-6. MR2944090

[11] G. L. EYINK and K. R. SREENIVASAN, Onsager and the theory of hydrodynamic turbulence, Rev. Modern Phys. 78 (2006), no. 1, 87-135. http://dx.doi.org/10.1103/RevModPhys. 78. 87. MR2214822

[12] B. GIDAS, W. M. NI, and L. NiRENBERG, Symmetry and related properties via the maximum principle, Comm. Math. Phys. 68 (1979), no. 3, 209-243. MR544879

[13] C. GUI, Al. JEVNiKAR, and A. MORADIFAM, Symmetry and uniqueness of solutions to some Liouville-type problems: Asymmetric sinh-Gordon equation, cosmic string equation and Toda system, preprint.

[14] D. HORSTMANN, Generalizing the Keller-Segel model: Lyapunov functionals, steady state analysis, and blow-up results for multi-species chemotaxis models in the presence of attraction and repulsion between competitive interacting species, J. Nonlinear Sci. 21 (2011), no. 2, 231-270. http://dx. doi.org/10.1007/s00332-010-9082-x. MR2788856

[15] Al. JEVNIKAR and W. YANG, Analytic aspects of the Tzitzéica equation: blow-up analysis and existence results, Calc. Var. Partial Differential Equations 56 (2017), no. 2, Art. 43, 38. http:// $\mathrm{dx}$.doi.org/10.1007/s00526-017-1136-6. MR3624939

[16] _ - A mean field equation involving positively supported probability measures: Blow-up phenomena and variational aspects, Proc. Roy. Soc. Edinburgh Sect. A 149 (2019), no. 2, 325-352. https://doi.org/10.1017/prm.2018.30. MR3937710

[17] J. JOST, G. WANG, D. YE, and C. ZHOU, The blow up analysis of solutions of the elliptic sinhGordon equation, Calc. Var. Partial Differential Equations 31 (2008), no. 2, 263-276. http:// dx.doi.org/10.1007/s00526-007-0116-7. MR2353873 
[18] N. I. KaVallaris, T. Ricciardi, and G. ZeCCA, A multi-species chemotaxis system: Lyapunov functionals, duality, critical mass, European J. Appl. Math. 29 (2018), no. 3, 515542, available at http://arxiv.org/abs/arXiv:1703.01636. http://dx.doi.org/10.1017/ S0956792517000286. MR3788454

[19] N. I. KAVALLARIS and T. SUZUKI, On the finite-time blow-up of a non-local parabolic equation describing chemotaxis, Differential Integral Equations 20 (2007), no. 3, 293-308. MR2293987

[20] C. S. LIN, An expository survey on the recent development of mean field equations, Discrete Contin. Dyn. Syst. 19 (2007), no. 2, 387-410. http://dx.doi.org/10.3934/dcds.2007.19. 387. MR2335753

[21] A. MALCHIODI, Topological methods for an elliptic equation with exponential nonlinearities, Discrete Contin. Dyn. Syst. 21 (2008), no. 1, 277-294. http://dx.doi.org/10.3934/dcds. 2008.21.277. MR2379466

[22] F. DE MARCHIS and T. RICCIARDI, Existence of stationary turbulent flows with variable positive vortex intensity, Nonlinear Anal. Real World Appl. 38 (2017), 222-244. http://dx.doi.org/ 10.1016/j .nonrwa. 2017.04.013. MR3670706

[23] J. MOSER, A sharp form of an inequality by N. Trudinger, Indiana Univ. Math. J. 20 (1970/71), 1077-1092. http://dx.doi.org/10.1512/iumj.1971.20.20101. MR0301504

[24] C. NERI, Statistical mechanics of the N-point vortex system with random intensities on a bounded domain, Ann. Inst. H. Poincaré Anal. Non Linéaire 21 (2004), no. 3, 381-399 (English, with English and French summaries). http://dx.doi.org/10.1016/ S0294-1449(03)00053-2. MR2068307

[25] H. OHTSUKA, T. RICCIARDI, and T. SUZUKI, Blow-up analysis for an elliptic equation describing stationary vortex flows with variable intensities in $2 D$-turbulence, J. Differential Equations 249 (2010), no. 6, 1436-1465. http://dx.doi.org/10.1016/j.jde.2010.06.006. MR2677801

[26] L. Onsager, Statistical hydrodynamics, Nuovo Cimento (9) 6 (1949), no. Supplemento, 2 (Convegno Internazionale di Meccanica Statistica), 279-287. MR0036116

[27] A. PISTOIA and T. RICCIARDI, Concentrating solutions for a Liouville type equation with variable intensities in 2D-turbulence, Nonlinearity 29 (2016), no. 2, 271-297. http://dx. doi .org/10. 1088/0951-7715/29/2/271. MR3461599

[28] - Sign-changing tower of bubbles for a sinh-Poisson equation with asymmetric exponents, Discrete Contin. Dyn. Syst. 37 (2017), no. 11, 5651-5692. http://dx.doi.org/10.3934/ dcds. 2017245. MR3681954

[29] A. Poliakovsky and G. Tarantello, On a planar Liouville-type problem in the study of selfgravitating strings, J. Differential Equations 252 (2012), no. 5, 3668-3693. http://dx. doi. org $/ 10.1016 / j \cdot j$ de.2011.11.006. MR2876669

[30] T. RICCIARDI and T. SUZUKI, Duality and best constant for a Trudinger-Moser inequality involving probability measures, J. Eur. Math. Soc. (JEMS) 16 (2014), no. 7, 1327-1348. http://dx . doi.org/10.4171/JEMS/462. MR3254327

[31] T. RICCIARDI and R. TAKAHASHI, Blow-up behavior for a degenerate elliptic sinh-Poisson equation with variable intensities, Calc. Var. Partial Differential Equations 55 (2016), no. 6, Art. 152, 25. http://dx.doi.org/10.1007/s00526-016-1090-8. MR3571203

[32] T. RiCCIARDi, R. TAKAHASHI, G. ZECCA, and X. ZHANG, On the existence and blow-up of solutions for a mean field equation with variable intensities, Atti Accad. Naz. Lincei Rend. Lincei Mat. Appl. 27 (2016), no. 4, 413-429. http://dx. doi.org/10.4171/RLM/741. MR3556339

[33] T. RICCIARDI and G. ZECCA, Blow-up analysis for some mean field equations involving probability measures from statistical hydrodynamics, Differential Integral Equations 25 (2012), no. 3-4, 201222. MR2917882

[34] Mass quantization and minimax solutions for Neri's mean field equation in 2D-turbulence, J. Differential Equations 260 (2016), no. 1, 339-369. http://dx.doi.org/10.1016/j.jde. 2015.08.045. MR3411675

[35] K. SAWADA and T. SUZUKI, Derivation of the equilibrium mean field equations of point vortex and vortex filament system, Theoret. Appl. Mech. Japan 56 (2008), 285-290. 
[36] I. SHAFRIR and G. WOLANSKY, The logarithmic HLS inequality for systems on compact manifolds, J. Funct. Anal. 227 (2005), no. 1, 200-226. http://dx.doi.org/10.1016/j.jfa.2004.09. 007. MR2165092

[37] J. SOPIK, C. SIRE, and P. H. CHAVANIS, Self-gravitating Brownian systems and bacterial populations with two or more types of particles, Physical Review E 72 (2005), no. 2, 026105. http: //dx. doi.org/PhysRevE. 72.026105.

[38] T. SUZUKI, Global analysis for a two-dimensional elliptic eigenvalue problem with the exponential nonlinearity, Ann. Inst. H. Poincaré Anal. Non Linéaire 9 (1992), no. 4, 367-397. http://dx. doi.org/10.1016/S0294-1449(16)30232-3. MR1186683

[39] __ Mean Field Theories and Dual Variation: Mathematical Structures of the Mesoscopic Model, 2nd ed., Atlantis Studies in Mathematics for Engineering and Science, vol. 11, Atlantis Press, Paris, 2015. http://dx.doi.org/10.2991/978-94-6239-154-3. MR3443959

[40] T. SUZUKI and Y. TOYOTA, Trudinger-Moser inequality for Boltzmann-Poisson equation with multi-intensities continuous case, Calc. Var. Partial Differential Equations 58 (2019), no. 2, Art. 77, 17. https://doi.org/10.1007/s00526-019-1520-5. MR3928760

[41] N. S. TRUDInger, On imbeddings into Orlicz spaces and some applications, J. Math. Mech. 17 (1967), no. 5, 473-483. http://dx.doi.org/10.1512/iumj .1968.17.17028. MR0216286

[42] Y. S. YANG, Self-duality of the gauge field equations and the cosmological constant, Comm. Math. Phys. 162 (1994), no. 3, 481-498. http://dx.doi.org/10.1007/BF02101744. MR1277473

TONIA RICCIARDI:

Dipartimento di Matematica e Applicazioni Università di Napoli Federico II

Via Cintia, Monte S. Angelo, 80126 Napoli Italy

E-MAIL: tonricci@unina.it

RYO TAKAHASHI:

Mathematics Education, Faculty of Education

Nara University of Education

Takabatake-cho, Nara-shi 630-8528

Japan

E-MAIL: r-takaha@nara-edu.ac.jp

KEY WORDS AND PHRASES: Liouville-type problems, optimal Moser-Trudinger constant, radial solutions.

2010 Mathematics Subject Classification: 35J47, 35A01, 35 B07.

Received: July 14, 2017. 